\title{
Pharmacist-Physician Collaboration at a Family Medicine Residency Program: A Focus Group Study
}

Keri Hager, PharmD, BCACP ; Courtney Murphy, PharmD ${ }^{1}$; Don Uden, PharmD, FCCP'; Brian Sick, $M D^{3}$

${ }^{1}$ Department of Pharmacy Practice and Pharmaceutical Sciences; University of Minnesota College of Pharmacy

${ }^{2}$ Department of Pharmaceutical Care and Health Systems; University of Minnesota College of Pharmacy

${ }^{3}$ Department of Internal Medicine; University of Minnesota Medical School

\begin{abstract}
Background: In response to transforming healthcare and pursuit of the Triple Aim, many health systems have added team members to expand the capabilities and effectiveness of the team to facilitate these aims. The objective of this study was to explore knowledge and perceptions of pharmacist-physician collaboration among family medicine residents (FMR), family medicine faculty (FMF), and pharmacist faculty and residents in a practice where clinical pharmacy services were relatively new. Understanding the nuances of pharmacist-physician interactions will provide insight into how to improve FMR education to prepare learners for patient-centered, teambased practice.

Methods: An exploratory descriptive qualitative study design was used to articulate perceptions of professional roles and team-based care in an interprofessional family medicine community-based clinical practice. Five, 60-minute focus groups were conducted in a clinical training setting that focuses on preparing family medicine physicians for collaborative rural primary care practice.

Results: Twenty-one FMRs, eight FMF, and six clinical pharmacists participated. Three themes emerged from the focus groups and were consistent across the groups: 1) roles of pharmacists recognized by physicians in different settings, 2) benefits to collaboration, and 3) keys to successful pharmacist-physician collaboration which include a) developing the relationship, b) optimizing communication, c) creating beneficial clinical workflow, d) clarifying roles and responsibilities, and e) increasing opportunities for meaningful interactions. Conclusion: This study demonstrated that by co-locating physicians and pharmacists in the same environment, and providing a basic structure for collaboration, a collaborative working relationship can be initiated. Practices looking to have more effective collaborative working relationships should strive to increase the frequency of interactions of the professions, help the physicians understand the abilities of pharmacists, and help the pharmacists articulate to the physicians the value of what they provide to patients. The five keys identified in this study are building blocks to advance a successful collaborative working relationship that positively benefits patient care and achieves the Quadruple Aim.
\end{abstract}

Key words: collaborative practice, pharmacist-physician collaboration, family medicine residency, team based primary care

\section{BACKGROUND}

The healthcare system in the United States has begun to focus on achieving the Triple Aim: improved health of populations, improved individual experience of care including outcomes and satisfaction, and reduced population per capita cost of care. ${ }^{1,2}$ Collaboration across professions in patient care plays a significant role in the success of the Triple Aim, and healthcare professionals must be properly trained to provide this collaborative care. ${ }^{1,2}$ While the Triple Aim addresses the outcomes of health system services, a revised version, the Quadruple Aim, also addresses the health of individuals who provide patient care. ${ }^{3}$ Studies show that burnout is high among physicians, with family medicine physicians experiencing one of the highest burnout rates in medical practice. ${ }^{4,5}$ Bodenheimer and Sinsky suggested using a team approach to the provision of patient care in order to better balance the physician's workload. ${ }^{3}$ Pharmacists, a relative newcomer to the outpatient clinical team, can positively impact patient care by performing some of the tasks historically done by physicians, which can alleviate physician burnout. ${ }^{6}$ When

Corresponding author: Keri Hager, PharmD, BCACP

Associate Professor, Director, UMD MTM Clinic

Phone: 218-726-6013; Email: khager@umn.edu pharmacists are a member of the primary care team, they provide benefits, such as answering drug information questions, communicating with the team about medication issues, increasing prescribing safety, and educating patients and providers. ${ }^{7-11}$ The literature also provides strong support for pharmacist-physician collaboration to improve health outcomes, as shown through significantly improved patient A1c, blood pressure, and asthma control after pharmacist interventions. ${ }^{12-18}$ When pharmacists are a part of the healthcare team, physicians see value in their services. ${ }^{7,8,11}$

While it is well understood that a pharmacist-physician collaboration model can improve health outcomes, the methods for fostering these collaborations is less clear. Brock and Doucette tested a model of collaborative working relationships between physicians and pharmacists, which shows that this collaboration goes through five stages. ${ }^{19}$ These stages start with professional awareness (stage 0), move through professional recognition (stage 1), exploration and trial (stage 2), and professional relationship expansion (stage 3 ), and end with a commitment to a collaborative working relationship (stage 4). However, the model was tested with practitioners who had been in practice an average of at least 14 years, and with pharmacists 
practicing in community pharmacies rather than co-located with physicians.

Interprofessional learning begins early in a health profession student's education and accelerates into graduate training as residents and finally as healthcare professionals. ${ }^{20}$ In Family Medicine, the Accreditation Council for Graduate Medical Education (ACGME) emphasizes the importance of team-based care. Residents are assessed on their understanding of roles and responsibilities of healthcare team members and their ability to engage in appropriate team-based care. ${ }^{21}$ Examination of this graduate level of training may provide a better understanding of how to improve pharmacist/physician collaboration. Understanding underlying knowledge and perceptions of pharmacist/physician collaboration may guide interventions to intentionally enhance collaboration. In the health sciences education literature, this is an area with limited published data. ${ }^{9}$ Additionally, there is little research about knowledge and perceptions of collaboration among pharmacists involved in Family Medicine residency programs.

The objective of this study was to explore knowledge and perceptions of pharmacist-physician collaboration among family medicine residents (FMR), family medicine faculty (FMF), and pharmacists $(P)$, including pharmacist faculty and pharmacy residents. This information will provide insight for improvement of FMR education to better prepare learners for patientcentered, team-based practice.

\section{METHODS}

\section{Research Design}

An exploratory, descriptive, qualitative study design was used to articulate perceptions of professional roles and team-based care in an interprofessional family medicine community-based clinical practice.

\section{Setting}

The study took place from January 8 to May 11, 2015 at the Duluth Family Medicine Residency Program (DFMRP), an affiliate of the University of Minnesota School of Medicine Department of Family Medicine and Community Health located in Duluth, Minnesota. DFMRP's goal is to prepare family medicine physicians for collaborative rural primary care practice. ${ }^{22}$ This primary care practice is comprised of family medicine faculty (including behavioral health and social work faculty), family medicine residents (first year, G1; second year, G2; third year, G3), ambulatory clinical pharmacists, and rotating pharmacy residents. The clinical pharmacists had been providing comprehensive medication management services in the clinic for 2.5 years at the time the study took place. ${ }^{23}$ The care team also includes nurses, medical assistants, and other clinic staff. The family medicine residents have progressively more time in the clinic, ranging from two to four half-days per week, and see an average of six patients per half-day. Team huddles occur prior to each shift, and all health professionals are co-located in a team area with each profession available for care conferences.

\section{Participants}

Given the study objective to explore knowledge and perceptions of pharmacist-physician collaboration, the investigators used purposive sampling and selected the following providers affiliated with DFMRP: FMR (G1, n=8; G2, n=10; G3, n=9), FMF (physicians, $n=6$; behavioral health practitioners, $n=2$ ), ambulatory clinical pharmacists from the clinic $(n=2)$, and pharmacy residents from the clinic $(n=2)$ and hospital $(n=2)$ where the FMRs interacted. FMR trainees and their faculty, as well as pharmacists, were included to get diverse perspectives of pharmacist-physician collaboration in the clinic. The ambulatory clinical pharmacists and pharmacy residents were combined into one group because of their small number and because they all provide comprehensive medication management services. In addition, the authors reasoned that medical residents with more exposure to clinical pharmacy services (i.e., residents further along in their training) may have differing opinions about pharmacist-physician collaboration than earlier learners. Therefore, the FMR focus groups were divided into G1, G2, and $\mathrm{G} 3$ resident cohorts.

\section{Interview Guide}

An interview guide comprised of seven questions and corresponding probes was developed by one of the authors $(\mathrm{KH})$ to facilitate discussions about knowledge and perceptions of professional roles and team collaboration. Prior to using the interview guide, the questions and probes were reviewed by members of an interprofessional education advisory board that was comprised of the residency program director and the two ambulatory clinical pharmacists. This board was chosen to provide critical feedback because of their clinical expertise, interest in understanding FMR perceptions of collaboration with pharmacists, and support of enhanced collaboration between physicians and pharmacists. Several of the interview questions were tailored specifically for pharmacists. For example, the question "What is the pharmacist's role/responsibility in teambased care?" used with the FMRs and FMFs was reworded to "What are your roles/responsibilities in team-based care?" when speaking to the pharmacists. The interview guide is provided in Appendix A.

\section{Data Collection Procedure}

Five, 60-minute focus groups were conducted for this study: (1) FMR - G1, (2) FMR - G2, (3) FMR - G3, (4) FMF, and (5) ambulatory clinical pharmacists and pharmacy residents. The FMR and FMF focus groups were conducted in the Family Medicine Clinic during a regularly scheduled one-hour meeting, whereas the pharmacist group was conducted at another primary care clinic site within the health system. Participants were invited to participate either by the residency program director (physician groups) or the ambulatory clinical pharmacist (pharmacist group) in person or by email. Focus groups were facilitated by one author $(\mathrm{KH})$, who 
was not part of the clinical site, but was known by some of the participants through her work with their interprofessional education advisory group. Focus groups were audio recorded and transcribed verbatim. A pharmacy student took notes during each interview to supplement the transcript data. This research was approved by the University of Minnesota Institutional Review Board and was exempt from full review.

\section{Data Analysis}

Content analysis of transcripts was conducted by the four investigators using a classic analysis strategy. ${ }^{24}$ Coding of the transcripts was completed manually in three steps. In the first step, two authors $(\mathrm{KH}, \mathrm{CM})$ independently coded the transcripts and came to consensus on codes and categories through discussion. Once consensus was achieved, a third author (DU) independently coded the transcripts. The three coders then arrived at consensus on codes and categories through discussion. To strengthen the rigor of the data analysis, the investigators invited a physician (BS) to code all of the data independently. Following this independent analysis, all four coders came to consensus on the main themes and subthemes that emerged from the data. The coders started with open coding of the text and then proceeded to develop categories using axial coding. ${ }^{25}$ For example, the open codes of "pharmacist role-medication reviews (clinic)," "pharmacist role-medication adherence (clinic)," and "pharmacist role-prevention (clinic)," were later merged into an overarching category called "pharmacist roleclinic." A multistage data analysis procedure was implemented to ensure that no new themes emerged from the data and theoretical saturation was achieved for each of the identified themes and subthemes. ${ }^{26}$ The study participants did not review any of the transcripts; however, a summary of findings and recommendations was presented to the residency program director and the interprofessional education advisory group members for their review and feedback. This step was added to assess the credibility and overall trustworthiness of the study findings. ${ }^{27}$

\section{RESULTS}

Twenty-one out of 27 family medicine residents ( $7 \mathrm{G} 1,8 \mathrm{G} 2,6$ G3), 8 family medicine faculty, and 6 pharmacists participated in the focus groups. The analysis of the focus groups yielded three primary themes and corresponding subthemes (Table 1). Details of the themes and supporting quotes are provided below. Attribution for each of the quotes is given as FMF (family medicine faculty), FMR (family medicine resident) and $P$ (pharmacist). The FMRs are listed by year of residency G1-G3. The individual participants are designated by $\mathrm{M}$ (male) or $\mathrm{F}$ (female) and number.

\section{Theme 1: Roles of pharmacists in different settings were recognized by physicians}

The physician's view of the pharmacist's role varied based on the setting - clinic, hospital, or community. Additionally, the way that the physician interacted and communicated with the pharmacist in each of those settings varied.

\section{Clinic}

FMRs and FMF described clinical pharmacy services in the outpatient clinic as a comprehensive review of medications, including preventive items (e.g., immunizations due). FMRs expressed that pharmacists in the outpatient clinic have a substantial role in patient medication adherence. While the pharmacists conduct comprehensive medication management, it is not clear if the FMRs and FMF understand that this is more than medication review. Interaction with the pharmacist in the clinic occurred primarily out of convenience. If a FMR was in the clinic and no clinical pharmacist was present, the FMR telephoned a pharmacist at the hospital on the floor or in the central pharmacy.

"If I have a patient that's on more than ten medicines I think that they should meet with the pharmacist and just go over it." G1 FMR M1

"If they're [pharmacists] not here, I have called the inpatient pharmacy before." G2 FMR F2

\section{Hospital}

FMRs and FMF expressed that inpatient pharmacists have a significant role in medication safety and answering medicationrelated questions. When in the hospital, FMRs reported that the pharmacist contacts the physician regarding medication safety issues, and the FMRs contact the pharmacist for medicationrelated questions.

"Inpatient [I] ask them to assist doing [a] drug interaction search or help with toxic zones or specifically dosing." FMF M4

"The pharmacists specifically are a nice safety net." G1 FMR F2

\section{Community}

FMRs knew the least about community pharmacy practice. For most, their only communication with community pharmacists typically addressed insurance not covering a medication. FMF thought that more collaborative relationships with community pharmacists existed in rural locations because the physicians and pharmacists in the small community know each other personally. FMRs reported that most of the communication with community pharmacists came from the pharmacist to the FMR and was intended to clarify prescription orders.

"What the community retail pharmacists are really useful for is [insurance] coverage issues." G2 FMR M1

"The community pharmacists are really on the other end of the phone and that's really unfortunate. You don't have any relationship or talk to them." FMF M2 
Theme 2: Physicians' perceived benefits to collaboration

The FMF and FMRs described multiple benefits of collaboration. These benefits included time efficiency, patient care safety, and provision of education to the FMRs.

\section{Time Efficiency}

FMRs expressed appreciation for time efficiency of their patient clinic visits after the pharmacist had seen the patient immediately prior.

$$
\begin{aligned}
& \text { "If they see the patient before me, that's really helpful } \\
& \text { because then I can say, 'here's five things that I should } \\
& \text { touch [on] today." G2 FMR M2 }
\end{aligned}
$$

\section{Patient Care Safety}

FMRs and FMF reported patient care was safer when pharmacists were involved, particularly in the hospital setting.

"[The pharmacists have] more of a presence and it's made the hospital a safer place because there are lots of medication errors and pharmacists tend to double check." FMF M4

\section{FMR Education Provided by Pharmacists}

Didactic education provided by pharmacists was considered high quality, relevant, and well-delivered. Educational efforts by FMF and clinical pharmacists/residents were targeted to the FMR learners. FMR and FMF reported pharmacists were a helpful resource who knew about both preventive health issues (e.g., immunizations) and specific medication-related issues (e.g., dosing, entering medication orders in the electronic health record).

"Their depth and breadth of knowledge, their willingness to dig deep into a topic, their willingness to teach. In our residency program, we have a chronic disease management curriculum, and the pharmacists have been integral in that process." FMF F1

"The pharm lectures are probably the highest yield lectures that we have." G2 FMR M2

However, there was not a reciprocal benefit to the pharmacists.

"I didn't really feel like we get a whole lot of education from them." P F4

Pharmacists providing education to the FMRs about the roles and responsibilities of the pharmacist on the team was seen as important by the FMRs and FMF. Most FMRs and FMF favored the idea that the FMRs need to understand what and how pharmacists do their work because if certain clinical pharmacy services are not available at future practice sites, FMRs should have the capability to quickly learn these skills, consult colleagues when necessary, and even advocate for practice change to include clinical pharmacy services.
"Sometimes the residents are over reliant on the pharmacists to, for example, come up with an antibiotic dosing regimen." FMF F4

Theme 3: Keys to successful pharmacist-physician collaboration Successful collaborations between pharmacists and physicians were described in five domains: a) develop the relationship, b) optimize communication, c) create beneficial clinical workflow, d) clarify roles and responsibilities, and e) increase opportunities for meaningful interactions.

\section{Develop the Relationship}

The key components to developing an effective collaborative relationship that emerged from all groups included trust, respect, previous experiences, and access/availability

"I think mutual respect, too. If there's ever any pointing fingers between one or the other about something wrong that's starting off [on the wrong foot]." G3 FMR F4

\section{Optimize Communication}

FMRs would like access to a pharmacist they trust 24 hours a day and seven days a week, especially where they can communicate face-to-face in real time. They were not always certain how to access clinical pharmacy services (ambulatory or inpatient), when a pharmacist was not on site in the clinic. There was variability in the means and frequency with which FMRs access clinical pharmacy services (phone, electronic health record, email), although face-to-face seemed to be the preferred mode. If a pharmacist was not available to speak face-to-face, FMRs would contact pharmacists with whom they were familiar. The FMF also spoke to the value of having everybody in the same room at the same time and how that facilitated the ability to effectively communicate. They expressed a desire for this to be true in all settings where they interact with pharmacists.

"The best thing we've done collaboratively is to actually put our interdisciplinary team in the same space." FMF F3

"I think definitely working side by side in the hospital or clinic makes a big difference." G2 FMR M2

\section{Create Beneficial Clinical Workflow}

FMRs, FMF, and pharmacists thought the best model of practice was for the pharmacist to see the patient in the clinic before the FMR. The pharmacists captured a comprehensive medication list and provided recommendations to the FMR immediately before the FMR saw the patient. FMRs appreciated that pharmacists maintained the FMR-patient relationship by providing only education to the patients and making their recommendations about medication changes directly to the FMR rather than to the patient. This communication protocol helped to prevent unreasonable patient expectations. 
"The thing that's nice about it is that they'll discuss it with the patient, but the patient does not have the expectation that when I walk in the room that's the changes I'm going to make" G2 FMR F2

\section{Clarify Roles/Responsibilities}

FMRs and FMF recognized that roles of pharmacy residents and pharmacists overlap. In the hospital and clinic, they viewed pharmacy residents as transient pharmacists, but used their services in the same manner as their clinical pharmacist preceptors.

"They're used interchangeably because the pharmacy residents are fabulous." FMF F1

Additionally, they recognized that some pharmacist and FMR patient care roles overlap (e.g., preventative care, medication review, and patient education).

"[l am] impressed at how much clinical medicine and how many clinical guidelines the pharmacists know - even better than a lot of physicians." G2 FMR M3

No focus group participants clearly articulated a model for "team accountability" for patient care, but they recognized that if someone accepts the responsibility for a task, then they were accountable for the outcome.

"If you asked that to be done, they took the baton. They're dosing. They're responsible." FMF F1

\section{Increase Opportunities for Meaningful Interactions}

FMRs felt getting to know their pharmacy colleagues would facilitate understanding roles and responsibilities and would promote collaboration. FMRs, FMF, and pharmacists described multiple ways to develop a collaborative relationship, including having inpatient and outpatient pharmacy residents, FMRs, and community pharmacists participate in resident orientation or inviting a community pharmacist to a noon conference and to see some patients together in clinic. FMF also suggested that pharmacists be faculty in the residency program and participate in faculty meetings.

\section{"A noon conference with a panel of small town pharmacists talking about how they work with physicians in those small communities." FMF F2 \\ "I think it would be really beneficial to have a group meeting in the very beginning of the residency year." P F2}

\section{DISCUSSION}

A summary of findings and recommendations was presented to the residency program director and the interprofessional education advisory group members for their review and feedback. As a result, several changes were developed and implemented to facilitate collaboration, including enhanced interprofessional orientation activities, creation of collaborative practice agreements, and opportunities for FMRs to take an elective rotation with a community pharmacist (Appendix B).

This study found that the pharmacist and physician groups, including residents, recognize the benefits of a collaborative care model, including time efficiency, patient care safety, and education of the medical residents. It was surprising that the perceived benefits to collaboration in this study were consistent across all levels of medical residents. The expectation would be that greater exposure over time would have increased the benefits of collaboration. Perhaps the level of collaboration is fixed, which limits the opportunities for enhanced benefits with increased exposure.

There are, however, opportunities to improve the level of collaboration between pharmacists and physicians. Based on Brock and Doucette's five stages of collaborative working relationships, the pharmacist-physician groups are working at a mid-stage. ${ }^{19}$ The two professions have moved beyond an awareness of each other (stage 0 ), and have built a foundation of trust (stage 1), within the clinic setting, and are now beginning to assess the pharmacist's clinical abilities within that clinical setting (stage 2). Except in the case of questions specifically about medication management or for referral for disease-specific interventions, such as anticoagulation management, there is not yet interdependence on one another (stage 3 ). The pharmacist is still the primary initiator of clinical partnerships. ${ }^{19}$ To fully move to the final stage, Commitment to Collaborative Working Relationship (stage 4), the physicians would have to see that the benefits of collaboration outweigh any risks and that there is consistent input and physical pharmacist presence. ${ }^{19}$ This study identified five keys to a successful collaboration between pharmacists and physicians, which could assist in moving resident physicians and pharmacists to higher stages of collaboration. These include developing relationships, optimizing communication, creating beneficial clinical workflow, clarifying roles and responsibilities, and increasing opportunities for meaningful interactions. There are many strategies for advancing these keys to successful collaboration. The study participants mentioned such things as co-locating the two professions, inviting more people to noon conferences, and improving the ability to access each other's services at all times of the day. Pharmacists can also explicitly educate physicians about what occurs in a medication management visit, the types of patients that could benefit, and provide clear instructions about how to refer patients for pharmacy services. ${ }^{9}$ Another intervention is to increase the frequency of face-to-face interactions, which will help to develop a relationship that increases trust between the professions. ${ }^{28,29}$ It is also important to emphasize how the work of the two professions can be complementary and reinforce the benefits of each other's expertise such that there is a win-win relationship. $^{19}$ 
A limitation of this study is that it occurred in only one site; research in additional sites may yield further insights. Additionally, there were not enough family medicine faculty from the different professions to analyze their data separately. The knowledge and perceptions of the pharmacy residents and faculty may vary among the faculty professions, and further research is warranted. Future research is needed to measure the impact of increased pharmacist-FMR collaboration on patient care outcomes, and also to explore collaboration with integrated behavioral health faculty and learners.

\section{CONCLUSION}

Enhanced collaboration will evolve and strengthen in a clinical environment where each team member knows the roles of each profession and uses those roles to optimize the way the professionals communicate, interact, and provide patient care. This study in a practice with relatively new clinical pharmacy services shows that by co-locating physicians and pharmacists in the same setting, and providing a basic structure for collaboration, a collaborative working relationship can be facilitated. Practices looking to have more effective collaborative working relationships should strive to increase the frequency of interactions of the professionals, help the physicians understand the abilities of pharmacists, and help the pharmacists articulate to the physicians the value of what they provide to patients. The five keys to successful collaboration identified in this study are building blocks to advance a robust collaborative working relationship that positively benefits patient care and achieves the Quadruple Aim.

\section{List of abbreviations}

- FMR: Family Medicine Resident

- FMF: Family Medicine Faculty

- G1: Post-graduate Year 1 Family Medicine Resident

- G2: Post-graduate Year 2 Family Medicine Resident

- G3: Post-graduate Year 3 Family Medicine Resident

- DFMRP: Duluth Family Medicine Residency Program

- Disclosures: None

Conflict of Interest: We declare no conflicts of interest or financial interests that the authors or members of their immediate families have in any product or service discussed in the manuscript, including grants (pending or received), employment, gifts, stock holdings or options, honoraria, consultancies, expert testimony, patents and royalties

Treatment of Human Subjects: IRB exemption granted

\section{References}

1. Berwick DM, Nolan TW, Whittington J. The triple aim: Care, health, and cost. Health Aff. 2008;27(3):759-769.

2. Earnest $M$, Brandt $B$. Aligning practice redesign and interprofessional education to advance triple aim outcomes. J Interprof Care. 2014;28(6):497-500.

3. Bodenheimer T, Sinsky C. From triple to quadruple aim: Care of the patient requires care of the provider. Ann Fam Med. 2014;12(6):573-576.

4. Spickard A, Gabbe SG, Christensen JF. Mid-career burnout in generalist and specialist physicians. JAMA. 2002;288(12):1447-1450.

5. Shanafelt TD, Boone S, Tan L, et al. Burnout and satisfaction with work-life balance among US physicians relative to the general US population. Arch Intern Med. 2012;172(18):1377-1385.

6. Bodenheimer TS, Smith MD. Primary care: Proposed solutions to the physician shortage without training more physicians. Health Aff. 2013;32(11):1881-1886.

7. Pottie K, Farrell B, Haydt S, et al. Integrating pharmacists into family practice teams: Physicians' perspectives on collaborative care. Can Fam Physician. 2008;54(12):1714-1715.e5.

8. Dolovich L, Pottie K, Kaczorowski J, et al. Integrating family medicine and pharmacy to advance primary care therapeutics. Clin Pharmacol Ther. 2008;83(6):913-917.

9. Lounsbery JL, Moon J, Humphrey A, Prasad S. Optimizing resident physician use of clinical pharmacy services. Fam Med. 2013;45(1):33-36.

10. Scott MA, Hitch B, Ray L, Colvin G. Integration of pharmacists into a patient-centered medical home. J Am Pharm Assoc. 2011;51(2):161-166.

11. Kucukarslan S, Lai S, Dong Y, Al-Bassam N, Kim K. Physician beliefs and attitudes toward collaboration with community pharmacists. Res Soc Adm Pharm. 2011;7(3):224-232.

12. Machado M, Bajcar J, Guzzo GC, Einarson TR. Sensitivity of patient outcomes to pharmacist interventions. Part I: Systematic review and metaanalysis in diabetes management. Ann Pharmacother. 2007;41(10):1569-1582.

13. Machado M, Bajcar J, Guzzo GC, Einarson TR. Sensitivity of patient outcomes to pharmacist interventions. Part II: Systematic review and metaanalysis in hypertension management. Ann Pharmacother 2007;41(11):1770-1781. 
14. Dickerson LM, Ables AZ, Everett CJ, et al. Measuring diabetes care in the national interdisciplinary primary care practice-based research network.

Pharmacotherapy. 2011;31(1):23-30.

15. Carter B, Clarke W, Ardery G, et al. A clusterrandomized effectiveness trial of a physician/pharmacist collaborative model to improve blood pressure control. Circ Cardiovasc Qual Outcomes. 2010; 3(4): 418-423.

16. Carter BL, Ardery G, Dawson JD, et al. Physician/pharmacist collaboration to improve blood pressure control. Arch Intern Med. 2009;169(21).

17. Gums TH, Carter BL, Milavetz G, et al. Physicianpharmacist collaborative management of asthma in primary care. Pharmacotherapy. 2014;34(10):10331042.

18. Doucette WR, McDonough RP, Klepser D, and McCarthy R. Comprehensive medication therapy management: Identifying and resolving drug-related issues in a community pharmacy. Clin Ther. 2005;27(7):1104-1111.

19. Brock KA, Doucette WR. Collaborative working relationships between pharmacists and physicians: an exploratory study. J Am Pharm Assoc. 2004;44(3):358365.

20. Institute of Medicine (US). Measuring the Impact of Interprofessional Education on Collaborative Practice and Patient Outcomes. Washington DC: The National Academies Press, 2015.

21. Accreditation Council for Graduate Medical Education. ACGME Program Requirements for Graduate Medical Education in Family Medicine. Updated June 11, 2017. http://www.acgme.org/Portals/0/PFAssets/ProgramR equirements/120_family_medicine_2017-07- 01.pdf?ver=2017-06-30-083354-350. Accessed Sep 25, 2017.

22. Duluth Family Medicine Residency Program. Our Program Overview. Duluth, MN. http://www.dfmrp.org/Main/Overview.aspx. Accessed July 15, 2015.

23. PCPCC: The Patient-Centered Medical Home: Integrating comprehensive medication management to optimize patient outcomes resource guide. Second edition. June 2012. https://www.pcpcc.org/sites/default/files/media/med management.pdf. Accessed February 18, 2018.

24. Krueger RA and Casey MA. Focus Groups: A Practical Guide for Applied Research. $5^{\text {th }}$ edition. Thousand Oaks, CA: SAGE Publications, Inc; 2015.

25. Corbin J and Strauss A. Grounded Theory Research: Procedures, Canons, and Evaluative Criteria. Qual Sociol. 1990;13(1): 62-114.

26. Cresswell JW, Roth CN. Qualitative inquiry and research design: Choosing among five traditions. Thousand Oaks, CA: Sage Publications; 2018.

27. Lietz CA and Zayas LE. Evaluating qualitative research for social work practitioners. Adv Soc Work. 2010;11(2):188-202.

28. Dow E, Hager KD, Cernohous T. Proximity of medication therapy management pharmacist to physician site may impact the degree of collaboration (BRIDGE Phase I). J Res Interprof Pract Educ. 2014;3(3).

29. Hager KD, Uden D, Tomaszewski D. Bridging the location gap: Physician perspectives of physician pharmacist collaboration in patient care (BRIDGE Phase II). J Res Interprof Pract Educ. 2015;5(2). 
Table 1. Main Themes and Subthemes

1. Roles of pharmacists in different settings were recognized by physicians

a. Clinic - medication therapy management and adherence

b. Hospital - medication safety and medication-specific questions

c. Community - medication coverage and dose clarification

2. Physicians' perceived benefits to collaboration
a. Time efficiency
b. Patient care safety
c. FMR education provided by pharmacists

3. Keys to successful pharmacist-physician collaboration
a. Develop the relationship
b. Optimize communication
c. Create beneficial clinical workflow
d. Clarify roles/responsibilities
e. Increase opportunities for meaningful interactions

Appendix A. Family Medicine Resident Focus Group Interviewer Guide

\begin{tabular}{|c|c|}
\hline $15 \mathrm{~min}$ & $\begin{array}{l}\text { (1) Who makes up the health care team? } \\
\text { PROBES: } \\
\text { Who are the team members? } \\
\text { What are their roles and responsibilities? }\end{array}$ \\
\hline $10 \mathrm{~min}$ & $\begin{array}{l}\text { (2) There are pharmacists that work here in the clinic. Describe what the pharmacists in } \\
\text { the clinic here do. } \\
\text { PROBES: } \\
\text { Describe how this compares with what the pharmacists in the hospital do. } \\
\text { How about in "retail" or community pharmacy? } \\
\text { What is the pharmacist's scope of practice? } \\
\text { What is the pharmacist's role/responsibility in team-based care? }\end{array}$ \\
\hline $10 \mathrm{~min}$ & $\begin{array}{l}\text { (3) Describe how you interact with the pharmacists in the clinic. } \\
\text { PROBE: } \\
\text { Describe how this compares with how you interact with pharmacists in the hospital or } \\
\text { retail/community. }\end{array}$ \\
\hline $10 \mathrm{~min}$ & (4) When and how is collaborative team-based care beneficial for patients? \\
\hline $10 \mathrm{~min}$ & (5) What do you expect from collaboration? \\
\hline $10 \mathrm{~min}$ & $\begin{array}{l}\text { (6) Describe how to develop collaborative relationships amongst patient care providers } \\
\text { from different professions. }\end{array}$ \\
\hline $10 \mathrm{~min}$ & $\begin{array}{l}\text { (7) Do you have ideas about additional collaborative opportunities with pharmacists to } \\
\text { enhance patient care? } \\
\text { PROBE: } \\
\text { Maybe something you've seen in other practice settings? }\end{array}$ \\
\hline
\end{tabular}


Appendix B. Practice and Education Changes in Response to Focus Group Results

- Clinical pharmacists are now family medicine residency (FMR) program faculty

- Enhanced orientation with first-year FMRs

o Team member roles/responsibilities modified team-based learning quiz

o Team care conference labeling/stereotype exercise

o Shadowing clinical pharmacists

o Group polypharmacy patient case review done by first-year family medicine residents and pharmacy residents together; case is facilitated by pharmacy faculty

- Implementation/utilization of Collaborative Practice Agreements (CPAs) at the residency program clinic:

o Developing a Type 2 Diabetes non-insulin CPA to be utilized with $3^{\text {rd }}$ year medical residents

o Opioid taper CPA*

o Tobacco cessation CPA

- Pharmacist Documentation

o Continue to write detailed but appropriate notes that are good for learning/teaching

- Pharmacist Workflow

o Continue/strive for back-to-back patient visits

o Conduct co-visits for observation/teaching/evaluating*

- Exposure to community pharmacy practice

o Opportunity for one FMR per month to shadow a community pharmacist

0 At this time, this is not required but considering making this a requirement in the future

- Opportunities for pharmacy residents to attend medical residency lectures/learning

o Invitations sent to pharmacy residents via email

- Set up a generic pharmacy resident access to New Innovations to access the lecture calendar*

- Drug Information Questions*

o Answers to medication questions posed to the pharmacy pool will be de-identified and shared with all residents for learning purposes

- Huddles*

o Occur at 8:15 AM and 1:15 PM on the second floor first and then first floor

o Pharmacists will attend

*Not yet fully implemented as of September 2017. 